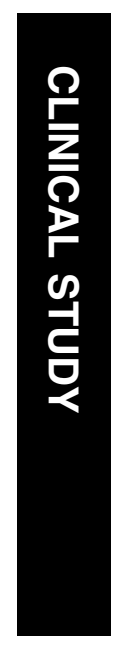

\section{Limited value of cyclosporine A for the treatment of patients with uveitis associated with juvenile idiopathic arthritis}

${ }^{1}$ Department of Ophthalmology, Uveitis Center, St Franziskus Hospital, Muenster, Germany

${ }^{2}$ German Center of Pediatric Rheumatology, GarmischPartenkirchen, Germany

${ }^{3}$ Department of Pediatric Rheumatology, St Josef Stift, Sendenhorst, Germany

${ }^{4}$ Department of Ophthalmology, Inselspital, University of Bern, Switzerland

Correspondence: A Heiligenhaus, Department of Ophthalmology, St Franziskus Hospital, Hohenzollernring 74 Muenster NRW 48145, Germany

Tel: + 49251933 080; Fax: + 492519330819 . E-mail: arnd.heiligenhaus@ uveitis-zentrum.de

Received: 1 October 2007 Accepted in revised form: 10 May 2008

Published online: 13 June 2008

Financial/proprietary interest: None

\begin{abstract}
Aims Juvenile idiopathic arthritis (JIA) is often associated with severe chronic anterior uveitis (CAU), and immunosuppressive therapy may be required. In this study, the value of cyclosporine $A$ (CsA) as monotherapy or as combination therapy for treating uveitis was studied in a large cohort of JIA children. Methods Multicentre retrospective study including 82 JIA children (girls $n=60$ ) suffering from unilateral or bilateral $(n=55)$ CAU. The indication for CsA was active uveitis, although patients were on topical or systemic corticosteroids, MTX, or other immunosuppressive drugs.

Results Inactivity of uveitis during the entire treatment period (mean 3.9 years) was obtained with CsA monotherapy in 6 of 25 (24\%) patients, but more often when CsA was combined with the immunosuppressives (35/ 72 patients; $48.6 \%, P=0.037)$, or MTX (18/37 patients, $48.6 \%, P=0.065)$, which had already been given. With CsA (mean dosage $2.9 \mathrm{mg} / \mathrm{kg}$ ), systemic immunosuppressive drugs and steroids could be reduced by $\geqslant 50 \%(n=19)$ or topical steroids reduced to $\leqslant 2$ drops/eye/day $(n=40)$ in selected patients. Pre-existing cystoid macular oedema did not resolve under CsA treatment in any of the patients. In nine patients (11\%), CsA was discontinued because of systemic hypertension $(n=1)$, elevated creatinine levels $(n=3)$, or other adverse effects $(n=5)$.

Conclusions These observations suggest that CsA has limited value as a second-line immunosuppressive drug for the treatment of JIA-associated CAU. The efficacy was better as the combination therapy in patients not
\end{abstract}

C Tappeiner ${ }^{1,4}$, M Roesel', C Heinz ${ }^{1}$, H Michels²,

$\mathrm{G} \mathrm{Ganser}^{3}$ and A Heiligenhaus ${ }^{1}$ responding to other immunosuppressives (eg, MTX) than the systemic monotherapy. Eye (2009) 23, 1192-1198; doi:10.1038/eye.2008.174; published online 13 June 2008

Keywords: childhood; immunosuppression; juvenile idiopathic arthritis; cyclosporine A; uveitis; iridocyclitis

\section{Introduction}

Juvenile idiopathic arthritis (JIA) comprises a heterogeneous group of rheumatoid diseases with the onset in children under 16 years of age and with an incidence of between four and 25 per 100000 of the population in Europe. ${ }^{1-3}$ The prevalence of uveitis in juvenile arthritis varies geographically, showing rates of between 10 and $18 \%$ in the United states of America and Scandinavia ${ }^{4}$ and $12 \%$ in Germany. ${ }^{5}$ Complications develop frequently, at rates of $25-50 \%$ in patients with JIA-associated uveitis. ${ }^{5-7}$ As the number of complications generally increases during the course of chronic disease, it is essential that inflammatory inactivity is achieved. A step-by-step antiinflammatory treatment approach is generally suggested, beginning with topical and possibly systemic corticosteroids. If quiescence cannot be achieved, one of the classical immunosuppressive drugs, eg, methotrexate (MTX), azathioprine (AZT) or cyclosporine A (CsA) is commonly used as a second-line medication. For many physicians, MTX is the first choice immunosuppressive, and CsA is often added to the regimen in patients who do not respond adequately to the treatment. 
Although CsA is frequently used for the treatment of JIA-associated uveitis, only very small case series with a total of 11 patients have been previously published on this specific issue. ${ }^{8-12}$ In the present report, we summarize our experience with CsA for the treatment of uveitis in 82 patients with JIA, with special reference to the value of CsA as systemic monotherapy and as a combination therapy in patients who do not respond to MTX. ${ }^{13}$

\section{Patients and methods}

A total of 82 children with confirmed JIA and associated chronic anterior uveitis (CAU) were analysed. The children were treated with CsA (Immunosporine ${ }^{\circledR}$, Sandimmune ${ }^{\circledR}$, or Neoral ${ }^{\circledR}$ ) for uveitis at the German Center of Pediatric Rheumatology in GarmischPartenkirchen (Germany), the Department of Pediatric Rheumatology, St Josef Stift Sendenhorst (Germany), or the Department of Ophthalmology at St Franziskus Hospital Muenster (Germany). The design of the work conforms to the standards currently applied in Germany. No institutional review board approval is required for the chart review studies.

The charts were reviewed retrospectively (monitoring period ranging from 1991 to 2006). In all patients, an infectious aetiology was excluded by negative serology for EBV, CMV, HSV, HZV, treponema, toxoplasma, toxocara, and negative tuberculosis skin test. The classification of juvenile arthritis was made in accordance with the current agreements of the International League of Associations for Rheumatology (ILAR). ${ }^{14}$

Rheumatologic examinations included a review of systems, laboratory tests, urine analysis, and chest X-ray, if necessary. Patients with uveitis unrelated to JIA were excluded, eg, intermediate, pars planitis, Fuchs heterochromic cyclitis, and infectious uveitis syndromes.

Ophthalmic evaluations included best-corrected visual acuities (BCVAs), slit-lamp examination, tonometry (Goldmann), and funduscopy.

Uveitis was classified anatomically in accordance with the Standardization of Uveitis Nomenclature (SUN) Working Group ${ }^{13,15}$ as anterior, intermediate, and posterior uveitis or panuveitis. Active uveitis was defined by the presence of $\geqslant 1+$ cells in the anterior chamber. Unilateral or bilateral disease and duration of uveitis were noted. Institution and termination of CsA therapy, CsA dosage, any adverse effects, any topical and anti-inflammatory medication before and under CsA therapy, the first uveitis manifestation in the second eye after institution of CsA therapy, and any uveitis-related complications in at least one eye were documented.

Patients were followed up at 6- to 12-month intervals both by the paediatric rheumatologist and ophthalmologist. Dosages of systemic corticosteroids and immunosuppressive agents were adjusted or drugs discontinued according to arthritis and uveitis activity and to the drug-related side effects.

The indication for systemic CsA monotherapy was active uveitis, despite the treatment with topical $(\geqslant 3$ drops/day) and possibly systemic corticosteroids ( $\geqslant 10 \mathrm{mg}$ or $\geqslant 0.15 \mathrm{mg} / \mathrm{kg}$ body weight). Sparing of systemic steroids was a major indication for CsA. Moreover, CsA was considered as a second- or third-line immunosuppressive, if uveitis remained active under treatment with any other immunosuppressives.

Inactivity or activity of uveitis under treatment at 1 year and during the entire treatment period was analysed. Inactive anterior uveitis was defined as rare cells or less in the anterior chamber. ${ }^{15}$ Additionally, the success of CsA therapy was analysed in accordance with a modified version of the grading system introduced by Saurenmann et al. ${ }^{16}$ The following outcome definitions for systemic treatment were used: good response, $\geqslant 50 \%$ decrease in both corticosteroid use and immunosuppressive agent (if not on combined immunosuppressive drug and systemic corticosteroid at study entry, then only $\geqslant 50 \%$ decrease of corticosteroids); moderate response, $\geqslant 50 \%$ decrease in either corticosteroid or immunosuppressive agent; and poor response, decrease of $<50 \%$ in both corticosteroid and immunosuppressive agent. Systemic and topical steroid sparing was analysed separately in our study.

For statistical analysis SPSS software (SPSS for Windows, version 11; SPSS Chicago, IL, USA) was employed. The $\chi^{2}$ square test and fisher's exact test for categorical data were used for statistical analysis when appropriate. A $P$-value $<0.05$ was considered as significant difference.

\section{Results}

Our analysis is based on the data derived from 82 children (137 eyes with uveitis) in whom CsA for the treatment of active uveitis was initiated in the period between 1991 and 2006 in the study centres. All of the patients had CAU that was associated with JIA. The epidemiological data and JIA subgroups are summarized in Table 1. The present regimen for systemic immunosuppression is described in Table 2.

The mean follow-up/treatment time after starting cyclosporine therapy was 3.9 years (range: 1.0-12.0). The initial CsA dosage was $3.0 \mathrm{mg} / \mathrm{kg}$ (range: $2.0-5.0 \mathrm{mg} / \mathrm{kg}$ ), and the mean dosage at the end of treatment or the end of the follow-up, if CsA was continued, was $2.8 \mathrm{mg} / \mathrm{kg}$ / day (range: 1.5-5.0). 
Table 1 Epidemiological data before the treatment with cyclosporine A in children with uveitis that is associated with juvenile idiopathic arthritis (JIA). $(n=82)$

\begin{tabular}{|c|c|c|c|}
\hline Female/male & $60(73.2 \%) / 22(26.8 \%)$ & & \\
\hline $\begin{array}{l}\text { Mean age at the onset of } \\
\text { arthritis (years) }\end{array}$ & 3.83 (range: 1-16) & & \\
\hline $\begin{array}{l}\text { Mean age at the onset of } \\
\text { uveitis (years) }\end{array}$ & 5.33 (range: 1-17) & & \\
\hline $\begin{array}{l}\text { Mean duration of } \\
\text { uveitis before study (years) }\end{array}$ & 4.77 (range: $0-21$ ) & & \\
\hline Active chronic anterior uveitis & $82(100 \%)$ & & \\
\hline JIA & $82(100 \%)$ & & \\
\hline Unilateral/bilateral & $27 / 55$ & & \\
\hline Uveitis complications & $48(58.5 \%)$ & & \\
\hline Cataract & $25(30.5 \%)$ & & \\
\hline Glaucoma & $3(3.7 \%)$ & & \\
\hline Synechiae & $37(45.1 \%)$ & & \\
\hline Cystoid macular oedema & $4(4.9 \%)$ & & \\
\hline $\begin{array}{l}\text { Mean follow-up time } \\
\text { on CsA (years) }\end{array}$ & 3.9 (range $1-12.0$ ) & & \\
\hline JIA subgroup & ANA positive & $\mathrm{N}$ & $\%$ \\
\hline Oligoarthritis & 61 & 65 & 79.5 \\
\hline Polyarthritis & 9 & 13 & 15.6 \\
\hline Psoriatic arthritis & 0 & 1 & 1.2 \\
\hline Other arthritis & 2 & 3 & 3.7 \\
\hline
\end{tabular}

ANA = antinuclear antibodies; CsA $=$ cyclosporine A.

JIA subgroups are classified according to the ILAR classification.

Table 2 Cylcosporine A for the treatment of 82 JIA patients with active chronic uveitis. Systemic immune-modulating therapy given before the initiation of cyclosporine A

\begin{tabular}{lc}
\hline Immunosuppressive agents & Number of patients (\%) \\
\hline No others & $21(25.6 \%)$ \\
Methotrexate & $45(54.9 \%)$ \\
Azathioprine & $15(18.3 \%)$ \\
Prednisone & $13(15.9 \%)$ \\
Adalimumab & $2(2.4 \%)$ \\
Etanercept & $1(1.2 \%)$ \\
Leflunomid & $1(1.2 \%)$ \\
\hline
\end{tabular}

\section{CsA as systemic immunosuppressive monotherapy}

CsA monotherapy was attempted in 25 patients (Figure 1, Table 3). Whereas the inactivity of uveitis was achieved in six of these patients $(24.0 \%)$, uveitis recurred or inflammation persisted in $19(76.0 \%)$. The gender $(P=0.81)$, age at the onset of arthritis $(P=0.1)$ or uveitis $(P=47)$, JIA subgroup $(P=92)$, ANA positivity $(P=0.5)$, bilateral uveitis $(P=0.45)$, and presence of complications at initial presentation $(P=0.66)$ had no significant influence on the response to treatment.
In two cases (2.0\%), the second eye was affected for the first time, although the patient was under treatment with CsA. The sparing effect of immunosuppressives and steroids differed markedly between the individuals (Table 3). Sixteen of the 25 patients developed new uveitis complications in at least one eye during the CsA monotherapy.

\section{CsA in systemic combined immunosuppressive therapy}

A total of 72 patients received CsA as part of a combined systemic immunosuppressive treatment regimen. In 57 patients, CsA was added to the current course of systemic immunosuppressives. In another 15 patients, a second systemic immunosuppressive agent was added when systemic CsA monotherapy proved to be unsuccessful (Figure 1).

Inactivity of uveitis was achieved in 32 of 72 patients $(44.4 \%)$ at 1 year $(P=0.49$ as compared to CsA monotherapy), and in 35 of 72 patients (48.6\%) during the entire period (mean 3.3 years) receiving CsA in combination with other systemic immunosuppressives (MTX $n=18$, AZT $n=11$, others $n=6$ ). Compared with the monotherapy, CsA was more effective when given as combination therapy $(P=0.037)$.

With respect to sparing of the other immunosuppressive and systemic steroid medication, the response to CsA treatment was good in 17, moderate in 12 , and poor in 43 of the 72 patients, according to the definition described above. ${ }^{16}$ In this respect, no significant difference was found between the CsA monoand combination therapy $(P=0.82)$. A sparing effect of topical steroids was obtained in 33 patients (reduction to $\leqslant 2$ drops/day $n=14$, tapered off $n=19$ ), while the dosage was unchanged in another 33, and even increased in one other patient (unknown dosage in four patients). These numbers did not differ significantly from those in the monotherapy group $(P=0.39)$.

\section{Cs $A$ added as second-line immunosuppressive drug in patients already on MTX}

As MTX is the most frequently used immunosuppressive in JIA-associated uveitis patients, the value of additional CsA was analysed, especially in those patients who were not responding to MTX. In 37 children, CsA was added as a second systemic drug, when uveitis remained active under the MTX therapy (Table 4). In 18 of the 37 children $(48.6 \%)$, uveitis inactivity was achieved during the entire treatment period and the difference compared to the respective effect with CsA monotherapy was close to the significance level $(P=0.065)$. An attempt to taper off MTX was made in 10 of these 18 children, but the uveitis recurred in seven of them, although they were receiving CsA. 


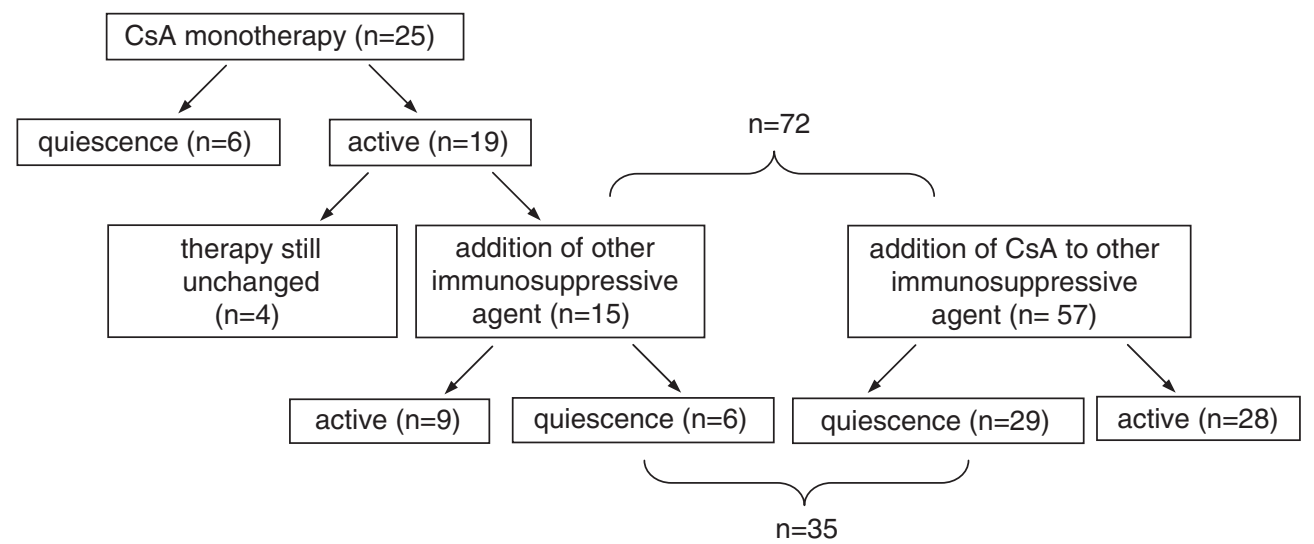

Figure 1 Uveitis response under CsA as monotherapy or as combination therapy with other immunosuppressive drugs. $\mathrm{CsA}=$ cyclosporine $\mathrm{A}, \mathrm{MTX}=$ methotrexate, $\mathrm{AZT}=$ azathioprine.

Table 3 Cylcosporine A (CsA) monotherapy as the initial line of immunosuppressive therapy of JIA patients with active chronic uveitis. Inactivity, sparing of anti-inflammatory medications and new uveitis complications under the treatment with CsA as a single systemic immunosuppressive drug, $n=25$ patients

Number of patients (\%)

$$
\text { At } 1 \text { year During the entire follow-up (mean } 2.0 \text { years) }
$$

\begin{tabular}{lcc}
\hline Inactivity of uveitis (Yes/no) & $9 / 16(36.0 / 64.0)$ & $6 / 19(24.0 / 76.0)$ \\
Sparing of anti-inflammatory medication as per a modified & definition of Saurenmann et $^{1 l^{16}}$ & \\
Good & $\mathrm{ND}$ & $5(20.0)$ \\
Moderate & $\mathrm{ND}$ & $2(8.0)$ \\
Poor & $\mathrm{ND}$ & $18(72.0)$ \\
& & \\
Sparing of topical steroids & $8(32.0)$ & $9(36.0)$ \\
Taper off & $7(28.0)$ & $6(24.0)$ \\
Reduced to $\leqslant 2$ drops /day & $8(32.0)$ & $8(32.0)$ \\
Unchanged & $2(8.0)$ & $3(12.0)$ \\
Increased & $\mathrm{ND}$ & $16(64.0)$ \\
Patients with new uveitis complications & &
\end{tabular}

JIA = juvenile idiopathic arthritis.

Table 4 Cylcosporine A (CsA) for the treatment of JIA patients with active chronic uveitis. Inactivity, sparing of anti-inflammatory medications and new uveitis complications under treatment with CsA when added as the second-line immunosuppressive drug in patients already on methotrexate; $n=37$ patients

Number of patients (\%)
At 1 year $\quad$ During entire follow-up (mean 2.9 years)

\begin{tabular}{|c|c|c|}
\hline Inactivity of uveitis (Yes/no) & $18 / 19(48.6 / 51.4)$ & $18 / 19(48.6 / 51.4)$ \\
\hline \multicolumn{3}{|c|}{ Sparing of anti-inflammatory medication as per a modified definition of Saurenmann et al ${ }^{16}$} \\
\hline Good & ND & $9(24.3)$ \\
\hline Moderate & ND & $8(21.6)$ \\
\hline Poor & ND & $20(54.1)$ \\
\hline \multicolumn{3}{|l|}{ Sparing of topical steroids } \\
\hline Taper off & $9(24.3)$ & $8(21.6)$ \\
\hline Reduced to $\leqslant 2$ drops / day & $11(29.7)$ & $12(27.0)$ \\
\hline Unchanged & $13(35.1)$ & $15(40.5)$ \\
\hline Increased & $1(2.7)$ & $2(5.4)$ \\
\hline Unknown & $3(8.1)$ & $2(5.4)$ \\
\hline Patients with new uveitis complications & ND & $17(45.9)$ \\
\hline
\end{tabular}

$\mathrm{JIA}=$ juvenile idiopathic arthritis. 
Table 5 Patients with ocular complications at the beginning and at the end of cyclosporine A (CsA) therapy; mean follow-up 3.9 years (range: $1-12$ )

\begin{tabular}{lcc}
\hline & $\begin{array}{c}\text { Before CsA therapy } \\
\text { Number of } \\
\text { patients (\%) }\end{array}$ & $\begin{array}{c}\text { Under CsA therapy } \\
\text { Number of } \\
\text { patients }(\%) \\
\text { at end of follow-up }\end{array}$ \\
\hline Any complications & $48(58.5)$ & $57(69.5)$ \\
Posterior synechiae & $37(45.1)$ & $41(50.0)$ \\
Cataract formation & $25(30.5)$ & $33(40.2)$ \\
Band keratopathy & $10(12.2)$ & $14(17.1)$ \\
Cystoid macular oedema & $4(4.9)$ & $6(7.3)$ \\
Glaucoma & $3(3.7)$ & $10(9.8)$ \\
Epiretinal gliosis & $3(3.7)$ & $4(4.9)$ \\
Dense vitreous opacities & $2(2.4)$ & $4(4.9)$ \\
Ocular hypotony & $2(2.4)$ & $2(2.4)$ \\
Retinal detachment & $0(0)$ & $2(2.4)$ \\
Phthisis & $1(1.2)$ & $1(1.2)$ \\
\hline
\end{tabular}

After adding CsA, immunosuppressive and/or systemic steroid sparing was possible in $45.9 \%$ of the patients. The systemic steroid or immunosuppressive sparing effect $(P=0.62)$ or sparing of topical steroids $(P=0.24)$ of additional CsA with MTX did not differ from the CsA monotherapy group.

\section{New complications under CsA}

Whereas 48 patients $(58.5 \%)$ already had developed uveitis complications before the initiation of CsA treatment, another nine patients $(10.9 \%)$ developed first uveitis complications under CsA (Table 5). As the extent of synechia formation was not determined, no conclusions can be drawn in this study on the worsening of the condition.

Sixteen of the 25 patients $(64 \%)$ developed new secondary complications during the treatment period with CsA monotherapy. New uveitis complications were reported in $35(48.6 \%)$ of the 72 patients under systemic combination immunosuppression therapy, and in 17 patients $(45.9 \%)$ with additional CsA to the MTX, and these numbers did not differ from the CsA monotherapy group ( $P=0.25$ and 0.2 , respectively).

Cystoid macular oedema (CME) had already been detected under the previous MTX therapy in four patients and did not disappear in any of the patients after adding CsA. Before CsA treatment, glaucoma was present in three patients and was documented in a total of 10 patients at the end of the follow-up.

\section{Side effects of $\operatorname{Cs} A$}

During the entire follow-up period, CsA treatment had to be stopped because of adverse reactions in $11 \%$ of all patients. Reported adverse reactions included systemic hypertension $(n=1)$, increase in serum creatinine level $(n=3)$, hypermennorrhoea $(n=1)$, periproctic abscess $(n=1)$, recurrent infections of the urinary tract $(n=1)$, and others $(n=2)$.

\section{Discussion}

Only little evidence has been published from a few patients to suggest that CsA may improve JIA-associated uveitis. However, the observations from 82 patients in this report reveal that this particular form of uveitis does not respond well to CsA as a monotherapy, and that MTX non-responders show better responses when CsA is added to the treatment regimen.

CsA binds to the cytosolic protein cyclophilin of lymphocytes, especially $\mathrm{T}$ lymphocytes. This inhibits calcineurin and reduces the transcription of interleukin (IL)-2. CsA reduces the production of various proinflammatory cytokines, eg, IL-2, -3 , interferon- $\gamma$, and tumour necrosis factor $-\alpha$, and inhibits the activation, proliferation, and function of effector T cells. For this reason, CsA has become widely established as a steroidsparing immunosuppressive, with proven effect for the treatment of uveitis in adults. ${ }^{17,18}$ CsA has also been introduced into the treatment of uveitis associated with JIA, although the role of T lymphocytes in the pathogenesis of JIA-associated uveitis has not been defined yet.

In their retrospective study, Kilmartin et $a l^{8}$ reported on 14 children suffering from uveitis who were treated with CsA. Inflammation improved at a dosage of $5 \mathrm{mg} / \mathrm{kg}$ in $76 \%$. Systemic steroids could be tapered off in four patients (29\%) and reduced in another 10 (71\%). Notably, only three of the patients had JIA and the response was not adequate in these children.

In a prospective, non-randomized study undertaken by Gerloni et al, ${ }^{10} 34$ JIA patients were treated with CsA at an initial average dosage of $4.2 \mathrm{mg} / \mathrm{kg} /$ day. However, uveitis was only present in seven of them. Twelve of 28 patients (43\%) with objective signs of arthritis showed an improvement in the number of active joints as compared to the baseline examination. Overall, the physicians' evaluations only indicated $24 \%$ improvement. In none of the seven uveitis patients could the steroid treatment be discontinued; the dose could be reduced in four patients, but had to be increased in another two. Uveitis activity was reduced in eight of the involved eyes $(66 \%)$ and visual acuity improved in $58 \%$ of them. Of the 34 patients 32 stopped taking CsA, of them $23 \%$ because of remission (none in the uveitis group), $43 \%$ because disease flared up or the treatment was ineffective (42\% in the uveitis group) and 26\% (none in the uveitis group) because of side effects. In 1 patient with uveitis not responding to combined MTX and prednisone, quiescence was 
achieved with additional CsA. The most common side effects from CsA were hypercreatinaemia (39\%), systemic hypertension (15\%) and hypertrichosis (29\%).

Schlote et $a l^{19}$ reported on 4 children with CAU who were treated with CsA. The 2 patients with JIA-associated uveitis responded only moderately to a combination of CsA and systemic prednisolone. All patients developed moderate hypertrichosis, and one had gingival hyperplasia.

Our observations suggest that CsA has limited efficacy in the treatment of JIA-associated CAU. During the mean follow up of 2.0 years under systemic CsA monotherapy, inactivity of uveitis was only maintained in 6 of 25 (24\%) children. After one year of treatment, uveitis was inactive in 9 of the 25 patients (36\%). And importantly, the treatment only produced a minor sparing effect of antiinflammatory medication. Although CsA is commonly used to treat uveitis in juvenile arthritis patients, our data suggest that its efficacy is low when administered as systemic immunosuppressive monotherapy in this patient population.

The impact of CsA on the visual outcome could not be determined in this study, as the documented VAs were frequently unreliable due to children's incompliance or opacities in the visual axis. Although this case series has been without a control group, the data reveal that new uveitis complications often occurred during the treatment period. Indeed, new complications were detected in 16 of the 25 patients $(64 \%)$ included, and in two of the patients, the first uveitis manifestation developed in the formerly uninvolved eye, although they were being treated with CsA. It is noteworthy that CME, which was present in four children before the initiation of CsA therapy, did not disappear under the treatment in any of them.

Previously, it has been reported that severe uveitis in juvenile arthritis could be controlled with a combination of CsA with MTX. ${ }^{7}$ The management of JIA children with uveitis, who are not responding to MTX is an important issue, as the drug is the most frequently used immunosuppressant in this patient group. It is, therefore, noteworthy that our data show that CsA was significantly more effective when it was added to an immunosuppressive drug, which had already been given. When CsA was added to MTX in the children with active uveitis, inactivity was achieved in approximately half of them. Our observations, however, show that the sparing effect of the other systemic immunosuppressive drugs and of topical steroids may be variable. For the entire patient series, systemic immunosuppressive drugs and steroids could be reduced by $\geqslant 50 \%$ in 19 patients, or topical steroids reduced to $\leqslant 2$ drops/eye/day in 40 patients.

Although we could speculate that the higher CsA dosages would have given better results in this study, the CsA dosages did not differ significantly between the responders and the non-responders. According to safety recommendations, the dosage for this patient group should be under $5 \mathrm{mg} / \mathrm{kg} /$ weight. It has been shown previously that at this dosage, nephrotoxicity can be kept to a minimum. . $11,20,21$ Therefore, alternative medication has been suggested, if treatment of 2 years or longer is expected. In our patients, the CsA dosage had, therefore, not exceeded $5 \mathrm{mg} / \mathrm{kg}$.

Apart from the anti-inflammatory value of these medications, drug safety is of the utmost importance in JIA children with uveitis, especially as long-term treatment is usually required. Thus, long-term safety data are required, which is a good reason for preferentially using particular drugs, eg, MTX, AZT, and also CsA. Therefore, the use of CsA may be favoured in patients who do not respond to MTX as contrasted to some recently introduced drugs, eg, TNF- $\alpha$ inhibitors, leflunomide, or mycofenolate mofetil.

Taken together, the present data suggest that the value of CsA in the step-ladder approach for the treatment of children with JIA-associated uveitis must be reconsidered. Although this study is somewhat limited by its retrospective design, the results suggest that CsA monotherapy is of limited value, while the addition of CsA may be beneficial in patients not responding properly to MTX or other immunosuppressive drugs. Prospective randomized studies and long-term registries are necessary to define the most effective and safe treatment regimen.

\section{References}

1 Mingels A, Hudde T, Heinz C, Heiligenhaus A. Vision-threatening complications in childhood uveitis. Ophthalmologe 2005; 102: 477-484.

2 Andersson Gare B. Juvenile arthritis - who gets it, where and when? A review of current data on incidence and prevalence. Clin Exp Rheumatol 1999; 17: 367-374.

3 Manners PJ, Bower C. World-wide prevalence of juvenile arthritis why does it vary so much? J Rheumatol 2002; 29: 1520-1530.

4 Carvounis PE, Herman DC, Cha S, Burke JP. Incidence and outcomes of uveitis in juvenile rheumatoid arthritis, a synthesis of the literature. Graefes Arch Clin Exp Ophthalmol 2006; 244: 281-290.

5 Heiligenhaus A, Niewerth M, Mingels A, Ganser G, Thon A, Pleyer $U$ et al. Epidemiology of uveitis in juvenile idiopathic arthritis from a national paediatric rheumatologic and ophthalmologic database. Klin Monatsbl Augenheilkd 2005; 222: 993-1001.

6 Edelsten C, Lee V, Bentley CR, Kanski JJ, Graham EM. An evaluation of baseline risk factors predicting severity in juvenile idiopathic arthritis associated uveitis and other chronic anterior uveitis in early childhood. Br J Ophthalmol 2002; 86: 51-56.

7 Paroli MP, Speranza S, Marino M, Pirraglia MP, Pivetti-Pezzi P. Prognosis of juvenile rheumatoid arthritis-associated uveitis. Eur J Ophthalmol 2003; 13: 616-621. 
8 Kilmartin DJ, Forrester JV, Dick AD. Cyclosporin A therapy in refractory non-infectious childhood uveitis. $\mathrm{Br} J$ Ophthalmol 1998; 82: 737-742.

9 Kotaniemi K. Late onset uveitis in juvenile-type chronic polyarthritis controlled with prednisolone, cyclosporin A and methotrexate. Clin Exp Rheumatol 1998; 16: 469-471.

10 Gerloni V, Cimaz R, Gattinara M, Arnoldi C, Pontikaki I, Fantini F. Efficacy and safety profile of cyclosporin A in the treatment of juvenile chronic (idiopathic) arthritis. Results of a 10-year prospective study. Rheumatology (Oxford) 2001; 40: 907-913.

11 Feutren G, Mihatsch MJ. Risk factors for cyclosporine-induced nephropathy in patients with autoimmune diseases. International Kidney Biopsy Registry of Cyclosporine in Autoimmune Diseases. N Engl J Med 1992; 326: 1654-1660.

12 Walton RC, Nussenblatt RB, Whitcup SM. Cyclosporine therapy for severe sight threatening uveitis in children and adolescents. Ophthalmology 1998; 105: 2028-2034.

13 Jabs DA, Nussenblatt RB, Rosenbaum JT. Standardization of uveitis nomenclature for reporting clinical data. Results of the first international workshop. Am J Ophthalmol 2005; 140: 509-516.

14 Petty RE, Southwood TR, Manners P, Baum J, Glass DN, Goldenberg $\mathrm{J}$ et al. International League of Associations for Rheumatology classification of juvenile idiopathic arthritis: second revision, edmonton 2001. J Rheumatol 2004; 31: 390-392.
15 Brooks P, Hochberg M. Outcome measures and classification criteria for the rheumatic diseases. A compilation of data from OMERACT (Outcome Measures for Arthritis Clinical Trials), ILAR (International League of Associations for Rheumatology), regional leagues and other groups. Rheumatology (Oxford) 2001; 40: 896-906.

16 Saurenmann RK, Levin AV, Rose JB, Parker S, Rabinovitch T, Tyrrell PN et al. Tumour necrosis factor alpha inhibitors in the treatment of childhood uveitis. Rheumatology (Oxford) 2006; 45: 982-989.

17 Towler HM, Cliffe AM, Whiting PH, Forrester JV. Low-dose cyclosporin A therapy in chronic posterior uveitis. Eye 1989; 3: $282-287$.

18 Vitale AT, Rodriguez A, Foster CS. Low-dose cyclosporin A therapy in treating chronic, noninfectious uveitis. Ophthalmology 1996; 103: 365-373; discussion 373-374.

19 Schlote T, Dannecker G, Thiel HJ, Zierhut M. Cyclosporin A in therapy of chronic uveitis in childhood. Ophthalmologe 1996; 93: 745-748.

20 Hoyer PF, Offner G, Oemar BS, Brodehl J, Ringe B, Pichlmayr R. Four years' experience with cyclosporin A in pediatric kidney transplantation. Acta Paediatr Scand 1990; 79: 622-629.

21 Tugwell P. International consensus recommendations on cyclosporin use in rheumatoid arthritis. Drugs 1995; 50: $48-56$. 